\title{
Combinatorial scoring auctions
}

Citation for published version (APA):

Müller, R. J., Perea ý Monsuwé, A., \& Wolf, S. (2007). Combinatorial scoring auctions. METEOR, Maastricht University School of Business and Economics. METEOR Research Memorandum No. 020 https://doi.org/10.26481/umamet.2007020

Document status and date:

Published: 01/01/2007

DOI:

10.26481/umamet.2007020

Document Version:

Publisher's PDF, also known as Version of record

\section{Please check the document version of this publication:}

- A submitted manuscript is the version of the article upon submission and before peer-review. There can be important differences between the submitted version and the official published version of record.

People interested in the research are advised to contact the author for the final version of the publication, or visit the DOI to the publisher's website.

- The final author version and the galley proof are versions of the publication after peer review.

- The final published version features the final layout of the paper including the volume, issue and page numbers.

Link to publication

\footnotetext{
General rights rights.

- You may freely distribute the URL identifying the publication in the public portal. please follow below link for the End User Agreement:

www.umlib.nl/taverne-license

Take down policy

If you believe that this document breaches copyright please contact us at:

repository@maastrichtuniversity.nl

providing details and we will investigate your claim.
}

Copyright and moral rights for the publications made accessible in the public portal are retained by the authors and/or other copyright owners and it is a condition of accessing publications that users recognise and abide by the legal requirements associated with these

- Users may download and print one copy of any publication from the public portal for the purpose of private study or research.

- You may not further distribute the material or use it for any profit-making activity or commercial gain

If the publication is distributed under the terms of Article $25 \mathrm{fa}$ of the Dutch Copyright Act, indicated by the "Taverne" license above, 
Rudolf Müller, Andrés Perea, Sascha Wolf

Combinatorial Scoring Auctions

$\mathrm{RM} / 07 / 020$

JEL code: C72, D44

\section{METE@R

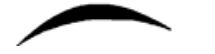

Maastricht research school of Economics

of TEchnology and ORganizations

Universiteit Maastricht

Faculty of Economics and Business Administration P.O. Box 616

NL - 6200 MD Maastricht

phone : ++31433883830

fax $\quad$ : ++31433884873 



\title{
Combinatorial Scoring Auctions
}

\author{
Rudolf Müller* $\quad$ Andrés Perea ${ }^{\dagger} \quad$ Sascha Wolf $\ddagger$
}

June 11, 2007

\begin{abstract}
This paper is concerned with a combinatorial, multi-attribute procurement mechanism called combinatorial scoring auction. In the setting that we analyze, private information of the suppliers is multi-dimensional. The buyer wants to procure several items at once. Subsets of these items are characterized by a price as well as by a number of non-monetary attributes called quality (e.g. completion time). The suppliers submit offers specifying prices and quality levels for these subsets. These offers are evaluated according to a quasilinear scoring rule. Based on the resulting scores suppliers win contracts for the delivery of certain items. Such a contract only specifies the set of items a supplier has to deliver and a score that he has to meet. The decision about the specific price-quality combination yielding this contracted score is at the discretion of the supplier who aims at optimizing his own profit.

We analyze the equilibria in such auctions and show the link between combinatorial scoring auctions and combinatorial price-only auctions. We demonstrate how this link can be used to employ preexisting knowledge about the equilibrium behavior in regular price-only auctions in the strategic analysis of combinatorial scoring auctions. Our results are the multi-item extension to the results of Asker and Cantillon (2007).
\end{abstract}

Keywords: Auction, Combinatorial, Multi-Attribute, Scoring Rule

JEL: C72, D44

${ }^{*}$ Department of Quantitative Economics, Maastricht University, P.O. Box 616, 6200 MD Maastricht, The Netherlands; e-mail: r.muller@ke.unimaas.nl

${ }^{\dagger}$ Department of Quantitative Economics, Maastricht University, P.O. Box 616, 6200 MD Maastricht, The Netherlands; e-mail: a.perea@ke.unimaas.nl

${ }^{\ddagger}$ Department of Quantitative Economics, Maastricht University, P.O. Box 616, 6200 MD Maastricht, The Netherlands; e-mail: s.wolf@ke.unimaas.nl 


\section{Introduction}

The design and analysis of combinatorial auctions is a flourishing field in auction theory drawing the interest of researchers from the area of game theory as well as from the area of computer science. (Or as Roger B. Myerson puts it on the back cover of Cramton, Shoham and Steinberg (2006): "Combinatorial auctions are the great frontier of auction theory today, ...".) While single item auctions only allocate one item at a time, combinatorial auctions are used to allocate multiple items all at once. By doing this, complementary or substitutable preferences of the bidders for different item sets can be taken into account. The majority of research conducted on combinatorial auctions (but this also holds for single item auctions) considers the price as the unique strategic dimension for bidders. That is, bidders offer prices for different sets of items based on which the items are allocated.

However, there are situations in which auction participants are not only concerned with the price of items but also with other non-monetary attributes of the items (called quality). Take for example the construction of a building. The construction process can be subdivided into several tasks on which contractors can bid. The building owner does not only care about the prices at which these tasks are executed by the contractors but also about non-monetary attributes of these tasks, like the completion time, the quality of the used materials, the construction quality or the probability that the contractor goes bankrupt during the job and leaves the task unfinished. On the other side, due to different levels of specialization, contractors might be able to take over several tasks or only some special tasks. Also, the contractor having the lowest cost for a specific task might differ depending on the quality level. That is, the low cost contractor for a task at a low quality level might not be the same as the low cost contractor for the same task at a higher quality level. Thus, it can be important to consider other strategic dimensions than just price.

One way to deal with combinatorial, multi-attribute procurement problems, like the one described above, is using combinatorial scoring auctions. In our paper we analyze the equilibria in such combinatorial scoring auctions. Specifically, we aim at extending the results of Asker and Cantillon (2007) who consider a setting for single item scoring auctions where item quality and bidders' types are multi-dimensional. For each bidder they construct a one-dimensional pseudo-type which is the maximum level of apparent social surplus that this bidder can generate ("apparent" because for its construction the scoring rule is used, which 
can differ from the buyer's true valuation function). Asker and Cantillon (2007) show that knowledge of bidders' pseudo-type distributions is sufficient for describing equilibrium outcomes and the buyer's expected equilibrium utility. This finding allows them to establish a link between single item scoring auctions and standard single item independent private value (IPV) price-only auctions. Specifically, for each single item scoring auction in their setting they identify a corresponding single item IPV price-only auction in which a bidder's type is his pseudo-type. They then show that the strategic analysis of a scoring auction reduces to the strategic analysis of the corresponding price-only auction. We establish the multi-item extension of this result. That is, we also construct pseudo-types, now consisting of the maximum levels of apparent social surplus that a bidder can generate for the different item sets, and we show a link between combinatorial scoring auctions and combinatorial price-only auctions. This link is again established by identifying for each combinatorial scoring auction a corresponding price-only auction.

\subsection{Related Work}

Che (1993) analyzes single item scoring auctions where the supplier bidding the highest score is contracted the delivery of the item. He considers a setting where the item quality is onedimensional, suppliers' private information is also one-dimensional and suppliers' production costs depend on the quality level as well as on their own private information. Furthermore, he considers scoring rules that are linear in price. In order to construct equilibria in these auctions he makes use of the maximum level of social welfare that a supplier can produce (which is well defined once the scoring rule is given), also called a supplier's pseudo-type. By employing a relatively simple change in variables, he is able to transform the problem of finding equilibria in the scoring auctions into the problem of finding equilibria in standard single item IPV price-only auctions, which are well-studied. He also designs an optimal scoring rule, maximizing the buyer's utility.

Branco (1997) extends Che's (1993) analysis of the independent cost setting to the case where suppliers' production costs are correlated. He characterizes an optimal direct revelation mechanism, the implementation of which requires a two-stage auction. David, AzoulaySchwartz and Kraus (2002a), (2002b), (2003) and (2006) propose and analyze simultaneous and sequential (with and without deadline) English auctions on suppliers' scores. Their 
settings allow for two-dimensional quality and two-dimensional private information, or general multi-dimensional quality and one-dimensional private information, respectively. However, in the settings that they consider the functional form of the suppliers' utility functions and the scoring rules are more restrictive than in the foregoing papers. They also identify optimal scoring rules for the considered settings.

Asker and Cantillon (2006), (2007) extend Che's (1993) analysis to a single item scoring auction setting that allows for multi-dimensional quality as well as multi-dimensional private information for the suppliers. Asker and Cantillon (2006) show that, unlike in Che's (1993) one-dimensional setting, the optimal buying mechanism cannot be implemented by a scoring auction with a scoring rule which is linear in price if suppliers information is multi-dimensional. Furthermore, they analyze the performance of scoring auctions in comparison to the optimal mechanism. Asker and Cantillon (2007) prove that in order to describe equilibrium outcomes in single item scoring auctions it is sufficient to make use of suppliers' one-dimensional pseudotypes. However, if suppliers' original private information is multi-dimensional, this proof is more involved than using the simple variable transformation employed by Che (1993). As Che (1993), Asker and Cantillon (2007) show that the problem of finding equilibria in single item scoring auctions can be transformed into the problem of finding equilibria in standard single item IPV price-only auctions. In this paper we deliver the multi-item extension of this result. Furthermore, Asker and Cantillon (2007) find that scoring auctions dominate other procedures for buying differentiated objects, like menu auctions, beauty contests and price-only auctions with minimum quality thresholds.

Milgrom (2000) shows that an item can be efficiently allocated by a scoring auction which employs the buyer's valuation function as the scoring rule and runs a Vickrey auction on suppliers' submitted scores. Suyama and Yokoo (2005) investigate a combinatorial multiattribute procurement auction setting that allows for multi-dimensional quality as well as for multi-dimensional private information of the suppliers. Their setting is a bit more restrictive than the one considered in this paper as the vector of quality levels for an item set is composed of the quality vectors of the individual items in the set. They propose a direct revelation Vickrey-Clarke-Groves (VCG) mechanism (Vickrey (1961), Clarke (1971), Groves (1973)) that determines the social surplus maximizing allocation of items and quality levels for the items. In this mechanism it is a dominant strategy for a supplier to report his private information 
truthfully. The combinatorial VCG scoring auction presented in Section 4.1 is quite similar in that it is essentially a direct revelation VCG mechanism based on suppliers' pseudo-types. In our auction the welfare maximizing allocation is determined by the auction mechanism while the optimal quality levels are determined by the suppliers themselves. This combinatorial VCG scoring auction is the multi-item extension of Milgrom's (2000) scoring auction.

In addition to the theoretical work presented above, also some experimental work on single item multi-attribute auctions has been done: Bichler (2000), Chen-Ritzo, Harrison, Kwasnica and Thomas (2005), Strecker and Seifert (2004), Bichler and Kalagnanam (2005). In these papers theoretical equilibrium predictions are verified and different scoring auction formats are compared. A finding of these experiments is that multi-attribute auction mechanisms dominate price-only auction mechanisms in terms of the buyer's and suppliers' utilities.

\subsection{Paper Outline}

In Section 2 we state some basic assumptions and definitions and describe our combinatorial scoring auction model which is an extension of the single item scoring auction models considered by Che (1993) and Asker and Cantillon (2007).

Contrary to the commonly analyzed single item scoring auctions, a buyer wants to procure several items at once in our setting. Item sets are characterized by a price as well as by a number of non-monetary attributes. A supplier's production costs for the different item sets are influenced by the chosen quality level and his type which is private information. We allow for multi-dimensional, independently distributed private information for the suppliers and multi-dimensional non-monetary attributes for the sets of items. The suppliers submit offers on the different item sets specifying prices as well as quality levels for the non-monetary attributes. Each offer is evaluated according to a scoring rule which assigns to each pricequality offer for an item set a one-dimensional score. ${ }^{1}$ We only consider scoring rules that are linear in price.

Based on the resulting scores an allocation rule determines which suppliers are contracted to deliver certain sets of items. This yields for each supplier an allocation vector which has an element for each item set (including the empty one) specifying the supplier's probability to win

\footnotetext{
${ }^{1}$ For an introduction to scoring rules and multi-attribute decision making in general see Yoon and Hwang (1995).
} 
the corresponding item set. (For example, in the case that the allocation rule is deterministic, one element of a suppliers allocation vector is equal to one, whereas the remaining elements are equal to zero.) The delivery contract for an item set specifies only a score that the winning supplier has to meet. The final decision about the specific price-quality combination for the delivered item set yielding this contracted score is at the discretion of the winning supplier. We find that winning suppliers choose the delivered quality level independent of the score that they contractually have to meet. That is, the contracted score effectively determines the price a winning supplier is charging the buyer for his item set. This finding is analogous to findings in single item scoring auction settings, see for example Che (1993) or Asker and Cantillon (2007).

In Section 2.2 we observe that, given the scoring rule, the maximum level of apparent social surplus that a supplier with a certain type can generate by producing and subsequently selling a set if items is well defined. We use this finding to construct for each supplier a pseudo-type which has an element for each set of items. Each of these elements is defined as the maximum level of apparent social surplus that the supplier can create for the corresponding item set. This definition of pseudo-types is analogous to the definition of one-dimensional pseudo-types in single item scoring auction settings (see Asker and Cantillon (2007)).

Based on the possible pseudo-types, we can partition a supplier's type space into equivalence classes of types yielding the same pseudo-type. A supplier's bidding function assigns a bid to each of his possible types. On a particular equivalence class, a supplier's bidding function is doing one of the following two things. Either the bidding function assigns the same bid to all types, in which case we say that it is not mixing on this equivalence class, or the bidding function assigns differing bids to the types, in which case we say that it is mixing on this equivalence class. This mixing can happen in two ways. If, given the bidding strategies of the others, differing bids yield also differing expected allocation vectors for the supplier then we speak of allocation mixing. However, if differing bids yield the same expected allocation vector then we speak of allocation equivalent mixing. Note that the bidding function can do both types of mixing on the same equivalence class.

In Section 3 we assume that in equilibrium suppliers' bidding functions employ allocation equivalent mixing only for a zero measure of types. Under this assumption we show (Theorem 1) that for the equilibrium analysis of a scoring auction it is sufficient to concentrate on bidding 
functions which are constant on equivalence classes of types, that is, they assign the same bid to all types yielding the same pseudo-type. Specifically, we show that for any equilibrium in a scoring auction we can construct a new equilibrium with bidding strategies that are constant on equivalence classes of types. A supplier's new bidding strategy is constructed by picking a representative type from each equivalence class in his type space and assigning the old equilibrium bids for these representative types also to all other types in their respective equivalence classes. In order to ensure that the new bidding strategies indeed constitute an equilibrium, it is sufficient to show that they differ from the suppliers' original equilibrium bidding strategies only on a set of types with zero measure (see proof of Theorem 1).

Unproblematic in this respect are the equivalence classes of types on which a supplier's original equilibrium bidding function is not mixing, since for these types his new bidding function is identical to his original one. Potentially troublesome are the equivalence classes on which the original bidding function is mixing, since for those types the new bidding strategy is differing from the original one. However, by assumption we have that the set of types for which the original bidding function employs allocation equivalent mixing has measure zero. In addition we prove (Lemma 4) that the set of types for which the original bidding function employs allocation mixing has also measure zero. ${ }^{2}$ This implies that the set of types for which the original bidding function employs mixing (that is, the union of the two aforementioned sets) has measure zero and hence, the new bidding strategy is almost everywhere the same as the original one.

Similar to Asker and Cantillon (2007) we use two main steps in order to establish Lemma 4 (stating that the set of types for which suppliers' equilibrium strategies employ allocation mixing has zero measure). First, we construct for every equilibrium in the scoring auction an auxiliary equilibrium (Lemma 2) in which suppliers' bidding strategies are based on their pseudo-types. This auxiliary equilibrium is constructed in such a way that a supplier's auxiliary equilibrium bidding strategy specifies a mixed bid for each of his pseudo-types. The support of such a mixed bid for a certain pseudo-type consists of all the pure bids that the original equilibrium bidding function specifies for types in the corresponding equivalence class.

\footnotetext{
${ }^{2}$ We have to exclude allocation equivalent mixing for a non-zero measure of types by assumption because we cannot exclude it analytically like in the case of allocation mixing. Asker and Cantillon (2007) consider only allocation mixing. Although they do not mention it explicitly, in order to derive their results, they also implicitly assume that in equilibrium allocation equivalent mixing is essentially excluded.
} 
In a second step we proof that for almost all pseudo-types this support does not contain pure bids that yield different expected allocations for the supplier (Lemma 3).

In Section 4 we show how the existing literature on combinatorial IPV price-only auctions can be used for the strategic analysis of scoring auctions, for example to establish equilibrium existence, revenue equivalence and so on. Specifically, for each scoring auction we identify a corresponding combinatorial price-only auction in which a bidder's type, on which he bases his bidding strategy, is his pseudo-type. By making use of the main result of the foregoing section (Theorem 1) we establish that every equilibrium in a scoring auction corresponds to an equilibrium in the related price-only auction which differs only on a zero measure of types, and vice versa, every equilibrium in the price-only auction is also an equilibrium in the related scoring auction (Corollary 1). This result is the multi-item analogue to Asker and Cantillon's (2007) finding that the equilibrium analysis of single item scoring auctions can be done by analyzing corresponding single item price-only auctions instead. With Corollary 2 we also extend Asker and Cantillon's (2007) "Expected Utility Equivalence"-Theorem for the buyer from single item to combinatorial scoring auctions.

In Section 4.1 we describe a combinatorial scoring auction that allocates items efficiently. As mentioned earlier in this section, this auction relates to the direct revelation VCG mechanism described by Suyama and Yokoo (2005) and is the multi-item extension of a single item scoring auction analyzed by Milgrom (2000). The above mentioned link to price-only auctions is used to establish an equilibrium in this auction.

\section{The Model}

A buyer wants to procure a set of distinct items $A=\{1, \ldots, a\}$. There is a set of potential suppliers $N=\{1, \ldots, n\}$. Each supplier is able to produce every item. ${ }^{3}$ The buyer does not need to purchase from only one supplier. He can buy different subsets of items from different suppliers. There are $2^{a}$ possible subsets of items, including the empty set which we associate with index 0. Each non-empty subset of items $j, j \in\left\{1, \ldots, 2^{a}-1\right\}$, is characterized by a sales price $p_{j} \in \mathbb{R}_{+}$and a quality level $q_{j} \in \mathbb{R}_{+}^{m_{j}}$ for its $m_{j} \geq 1$ non-monetary attributes. In

\footnotetext{
${ }^{3}$ In case a supplier is not able to produce certain items, this can be modeled by letting him bid infinitely high prices for the item sets that he is unable to produce. That way he is not chosen as the supplier for item sets that he cannot deliver.
} 
order to ease notation later on let us define $d=2^{a}-1$ and $m=\sum_{j=1}^{d} m_{j}$.

The buyer's valuation for the purchase of item set $j$ with quality level $q_{j}$ at price $p_{j}$ is $v_{j}\left(q_{j}\right)-p_{j}$. Each supplier $i$ has a type $\theta^{i} \in \Theta$ with $\Theta \subset \mathbb{R}^{k}$. $\Theta^{-i}$ denotes the set of all type profiles $\theta^{-i}=\left(\theta^{1}, \ldots, \theta^{i-1}, \theta^{i+1}, \ldots, \theta^{n}\right)$. Supplier $i$ 's type influences his production cost. The cost he incurs for producing the set of items $j$ with quality $q_{j}$ is denoted by $c_{j}\left(q_{j}, \theta^{i}\right)$. Note that all suppliers have the same cost function for item set $j .{ }^{4}$ We assume that $c_{j}$ is twice continuously differentiable as well as strictly increasing in both quality and his type. Supplier $i$ 's profit $\pi_{j}^{i}$ from producing the set of items $j$ with quality $q_{j}$ and selling it at price $p_{j}$ is

$$
\pi_{j}^{i}\left(p_{j}, q_{j}, \theta^{i}\right)=p_{j}-c_{j}\left(q_{j}, \theta^{i}\right)
$$

We assume that suppliers' type spaces are convex and compact. Types are privately observed and independently distributed. The probability densities are common knowledge. Let $\gamma^{i}$ denote supplier $i$ 's density on $\Theta$. The joint density $\gamma^{-i}$ on $\Theta^{-i}$ is then given by

$$
\gamma^{-i}\left(\theta^{-i}\right)=\prod_{h \neq i} \gamma^{h}\left(\theta^{h}\right)
$$

\subsection{The Scoring Auction}

The scoring auction works as follows. Based on his type each supplier $i$ makes a price-quality bid $\left(p^{i}, q^{i}\right) \in \mathbb{R}_{+}^{d+m}$ consisting of a price-quality offer for each of the possible non-empty item sets, that is $\left(p^{i}, q^{i}\right)=\left(\left(p_{1}^{i}, q_{1}^{i}\right), \ldots,\left(p_{d}^{i}, q_{d}^{i}\right)\right)$ with $\left(p_{j}^{i}, q_{j}^{i}\right) \in \mathbb{R}_{+}^{1+m_{j}} \cdot{ }^{5}$ There is a scoring rule

$$
S_{j}: \mathbb{R}_{+}^{1+m_{j}} \mapsto \mathbb{R}
$$

for every possible set of items $j$. Using this scoring rule, each price-quality offer $\left(p_{j}, q_{j}\right)$ for the item set $j$ is associated with a one-dimensional score $S_{j}\left(p_{j}, q_{j}\right)$. The scoring rules for all item sets are fixed before the bidding starts and known to every supplier. We assume that

\footnotetext{
${ }^{4}$ The assumption of suppliers having the same type spaces and the same cost functions is made for notational convenience and can easily be relaxed.

${ }^{5}$ We are aware of the potential complexity of suppliers' bids. Specifying a price-quality offer for each of the $2^{a}-1$ non-empty subsets of items is highly impractical for larger sets of items. Bid complexity and representation are generally an issue in combinatorial auctions. However, concerns about the representation of bids and bidding languages go beyond the scope of this paper and are not addressed henceforth. A comprehensive introduction to bidding languages for combinatorial auctions is given by Nisan (2006).
} 
the scoring rules are quasi-linear, that is

$$
S_{j}\left(p_{j}, q_{j}\right)=\phi_{j}\left(q_{j}\right)-p_{j}
$$

In addition it is assumed that $\phi_{j}$ is twice continuously differentiable and strictly increasing in $q_{j}$. Furthermore, we assume that for $\phi_{j}\left(q_{j}\right)-c_{j}\left(q_{j}, \theta^{i}\right)$ the matrix comprised of the second-order partial derivatives w.r.t. $q_{j}$ is negative definite. More specifically, for $g\left(q_{j}, \theta^{i}\right)=$ $\phi_{j}\left(q_{j}\right)-c_{j}\left(q_{j}, \theta^{i}\right)$ we assume that the matrix

$$
H\left(q_{j}, \theta^{i}\right)=\left(\begin{array}{ccc}
\frac{\partial^{2} g}{\partial q_{j, 1} q_{j, 1}}\left(q_{j}, \theta^{i}\right) & \cdots & \frac{\partial^{2} g}{\partial q_{j, 1} q_{j, m_{j}}}\left(q_{j}, \theta^{i}\right) \\
\vdots & \ddots & \vdots \\
\frac{\partial^{2} g}{\partial q_{j, m_{j}} q_{j, 1}}\left(q_{j}, \theta^{i}\right) & \cdots & \frac{\partial^{2} g}{\partial q_{j, m_{j}} q_{j, m_{j}}}\left(q_{j}, \theta^{i}\right)
\end{array}\right),
$$

where $q_{j, h}$ denotes the $h$ th element of $q_{j}$, is negative definite for all $q_{j}$ and $\theta^{i}$. Thus, $g$ is strictly concave in $q_{j}$ for all $\theta^{i}$.

By applying the scoring rules each supplier's original price-quality bid $\left(p^{i}, q^{i}\right) \in \mathbb{R}_{+}^{d+m}$ is transformed into a vector of scores $s^{i} \in \mathbb{R}^{d}$ consisting of a score $s_{j}^{i} \in \mathbb{R}$ for every set of items $j$. An allocation rule maps each profile of scores $s=\left(s^{1}, \ldots, s^{n}\right)$ into an allocation of items to suppliers (or a distribution over such allocations in case the allocation rule is probabilistic), that is it determines which supplier is allowed to provide which items to the buyer. Every item is provided by at most one supplier which allows for the case that some items are not procured at all. The allocation rule implies for each supplier $i$ and each profile of scores $s$ an allocation vector $x^{i}(s)=\left(x_{0}^{i}(s), x_{1}^{i}(s) \ldots, x_{d}^{i}(s)\right)$ where $x_{0}^{i}(s)$ denotes the probability that $i$ is awarded the empty set, that is he does not sell any items to the buyer. Similarly, for $j \in\{1, \ldots, d\}, x_{j}^{i}(s)$ denotes supplier $i$ 's probability for winning the contract giving him the right to provide the buyer with the set of items $j$. In order to simplify notation we define the allocation rule

$$
x: \mathbb{R}^{n d} \mapsto[0,1]^{n 2^{a}}
$$

directly as a mapping that maps each profile of scores $s \in \mathbb{R}^{n d}$ into an allocation vector $x^{i}(s) \in[0,1]^{2^{a}}$ for every supplier $i$.

Based on a profile of scores $s$, the winning score rule

$$
w: \mathbb{R}^{n d} \mapsto \mathbb{R}^{n 2^{a}}
$$


assigns each supplier $i$ with a vector of winning scores $w^{i}(s)=\left(w_{0}^{i}(s), w_{1}^{i}(s), \ldots, w_{d}^{i}(s)\right)$ where $w_{0}^{i}(s)$ is the payment that $i$ has to make in case he is awarded the empty set. Thus, we allow for the case that a supplier has to make a payment to the buyer if he did not win any delivery contract. For $j \in\{1, \ldots, d\}, w_{j}^{i}(s)$ is the score that supplier $i$ has to meet in case he wins the contract to provide the buyer with item set $j$. That is, he has to provide the set of items with a quality level $q_{j}$ at a price $p_{j}$ such that $S_{j}\left(p_{j}, q_{j}\right)=w_{j}^{i}(s)$. This means that the buyer and the supplier only contract a score for the item set. The actual sales price and the delivered quality is chosen by the supplier in such a way that the contracted score is met.

\subsection{Pseudo-types}

Suppose that supplier $i$ of type $\theta^{i}$ has won the contract to provide the set of items $j$ to the buyer and has to meet a score $\omega$. He will choose a price-quality pair $\left(p_{j}, q_{j}\right)$ that maximizes his profit $\pi_{j}^{i}\left(p_{j}, q_{j}, \theta^{i}\right)$ while meeting the score, that is $S_{j}\left(p_{j}, q_{j}\right)=\omega$. Using (1) and (2), his optimization problem becomes

$$
\begin{array}{ll} 
& \max _{\left(p_{j}, q_{j}\right)}\left(p_{j}-c_{j}\left(q_{j}, \theta^{i}\right)\right) \\
\text { s.t. } & \phi_{j}\left(q_{j}\right)-p_{j}=\omega .
\end{array}
$$

Substituting for $p_{j}$ in the objective function yields

$$
\max _{q_{j}}\left(\phi_{j}\left(q_{j}\right)-c_{j}\left(q_{j}, \theta^{i}\right)\right)-\omega
$$

As can be seen in (4), the supplier chooses the optimal quality level independent of the winning score that he has to meet. We assume that for every $\theta^{i} \in \Theta$ there exits a $q_{j}^{*}>0$ that maximizes $\phi_{j}\left(q_{j}\right)-c_{j}\left(q_{j}, \theta^{i}\right)$. Together with the strict concavity of $\phi_{j}\left(q_{j}\right)-c_{j}\left(q_{j}, \theta^{i}\right)$ in $q_{j}$ for all $\theta^{i}$ (see assumptions made earlier) this implies that $q_{j}^{*}$ is the only maximum and hence $\arg \max _{q_{j}}\left(\phi_{j}\left(q_{j}\right)-c_{j}\left(q_{j}, \theta^{i}\right)\right)$ is well defined. Furthermore, we find the following:

Lemma 1 The set $\left\{\max _{q_{j}}\left(\phi_{j}\left(q_{j}\right)-c_{j}\left(q_{j}, \theta^{i}\right)\right) \mid \theta^{i} \in \Theta\right\}$ is a closed interval in $\mathbb{R}$.

\section{Proof}

Define $g\left(q_{j}, \theta^{i}\right)=\phi_{j}\left(q_{j}\right)-c_{j}\left(q_{j}, \theta^{i}\right)$. Given the assumptions about $g\left(q_{j}, \theta^{i}\right)$ made earlier, we have that for each $\theta^{i}$ there exists a unique quality level $q_{j}^{*}$ that maximizes $g\left(q_{j}, \theta^{i}\right)$. Furthermore, we have that the first-order partial derivatives of $g$ w.r.t. $q_{j}$ are equal to zero 
at $q_{j}^{*}$. That is,

$$
\frac{\partial g}{\partial q_{j, 1}}\left(q_{j}^{*}, \theta^{i}\right)=\ldots=\frac{\partial g}{\partial q_{j, m_{j}}}\left(q_{j}^{*}, \theta^{i}\right)=0,
$$

where $q_{j, h}$ denotes the $h$ th element of $q_{j}$. From this and the assumptions about the secondorder partial derivatives of $g$ made earlier it follows by the Implicit Function Theorem ${ }^{6}$ that $q_{j}^{*}$ is a continuous function of $\theta^{i}$. Thus,

$$
\max _{q_{j}}\left(\phi_{j}\left(q_{j}\right)-c_{j}\left(q_{j}, \theta^{i}\right)\right)=\phi_{j}\left(q_{j}^{*}\left(\theta^{i}\right)\right)-c_{j}\left(q_{j}^{*}\left(\theta^{i}\right), \theta^{i}\right)
$$

is a continuous mapping from $\Theta$ to $\mathbb{R}$. Since $\Theta$ is compact and connected also its image under this mapping is compact and connected. This implies that the image is a closed interval in $\mathbb{R}$.

Based on the above we define for every $\theta^{i}$ in supplier $i$ 's type space

$$
t\left(\theta^{i}\right)=\left(\max _{q_{1}}\left(\phi_{1}\left(q_{1}\right)-c_{1}\left(q_{1}, \theta^{i}\right)\right), \ldots, \max _{q_{d}}\left(\phi_{d}\left(q_{d}\right)-c_{d}\left(q_{d}, \theta^{i}\right)\right)\right) .
$$

We call $t\left(\theta^{i}\right)$ the supplier's pseudo-type. Note that suppliers' pseudo-types are dependent on their types as well as on the chosen scoring rules and the production cost functions. Supplier $i$ 's pseudo-type is monotonically decreasing in his type: Take $\hat{\theta}^{i}, \theta^{i} \in \Theta$ such that $\hat{\theta}^{i}$ is componentwise smaller than $\theta^{i}$ and let $q_{j}^{* i}($.$) denote the optimal quality level that supplier$ $i$ picks for item set $j$ in (4) based on his type. Then, for each element $j$ of his pseudo-type,

$$
\begin{aligned}
t_{j}\left(\theta^{i}\right) & =\phi_{j}\left(q_{j}^{* i}\left(\theta^{i}\right)\right)-c_{j}\left(q_{j}^{* i}\left(\theta^{i}\right), \theta^{i}\right) \\
& <\phi_{j}\left(q_{j}^{* i}\left(\theta^{i}\right)\right)-c_{j}\left(q_{j}^{* i}\left(\theta^{i}\right), \hat{\theta}^{i}\right) \\
& \leq \phi_{j}\left(q_{j}^{* i}\left(\hat{\theta}^{i}\right)\right)-c_{j}\left(q_{j}^{* i}\left(\hat{\theta}^{i}\right), \hat{\theta}^{i}\right)=t_{j}\left(\hat{\theta}^{i}\right),
\end{aligned}
$$

where the first inequality follows from the fact that costs are increasing in the type, and the second inequality follows from the optimality of $q_{j}^{* i}($.$) .$

$\Theta_{t}$ denotes the set of all types $\theta^{i} \in \Theta$ yielding the same $t$ as pseudo-type, that is $\Theta_{t}=\left\{\theta^{i} \in \Theta \mid t\left(\theta^{i}\right)=t\right\}$. A supplier's pseudo-type space is denoted $T$ with $T \subset \mathbb{R}^{d}$. It is convex and compact. Pseudo-types are privately observed and independently distributed. The distributions are common knowledge (since the scoring rules, the production cost functions and suppliers' type distributions are common knowledge). For technical reasons we

\footnotetext{
${ }^{6}$ The Implicit Function Theorem can be found in a variety of books on advanced calculus, for example Adams (2003), p.769.
} 
make the additional assumption that the pseudo-type mapping has the following property: If a set of pseudo-types has Lebesgue measure zero then also the set of types yielding this set of pseudo-types has Lebesgue measure zero. ${ }^{7}$

Suppose that suppliers have types $\theta=\left(\theta^{1}, \ldots, \theta^{n}\right)$ and make reports $\left(p^{1}, q^{1}\right), \ldots,\left(p^{n}, q^{n}\right)$ implying the profile of scores $s=\left(s^{1}, \ldots, s^{n}\right)$. The utility that supplier $i$ derives from the resulting outcome $(x(s), w(s))$ is

$$
u^{i}\left(s \mid \theta^{i}\right)=\sum_{j=1}^{d} x_{j}^{i}(s)\left(\max _{q_{j}}\left(\phi_{j}\left(q_{j}\right)-c_{j}\left(q_{j}, \theta^{i}\right)\right)-w_{j}^{i}(s)\right)-x_{0}^{i}(s) w_{0}^{i}(s) .
$$

Defining $\tilde{x}^{i}(s)=\left(x_{1}^{i}(s), \ldots, x_{d}^{i}(s)\right)$ (that is, $\tilde{x}^{i}(s)$ is the allocation vector $x^{i}(s)$ without the winning probability for the empty set $\left.x_{0}^{i}(s)\right)$ and using (5) this can be written as

$$
u^{i}\left(s \mid \theta^{i}\right)=\tilde{x}^{i}(s) t\left(\theta^{i}\right)-x^{i}(s) w^{i}(s){ }^{8}
$$

Notice that supplier $i$ 's pseudo-type captures his preference over outcomes $(x(),. w()$.$) . (As$ shown by Asker and Cantillon (2007) only quasi-linear scoring rules exhibit this property if suppliers' types are multi-dimensional.) By defining

$$
y^{i}(s)=x^{i}(s) w^{i}(s)
$$

we can simplify $(6)$ to

$$
u^{i}\left(s \mid \theta^{i}\right)=\tilde{x}^{i}(s) t\left(\theta^{i}\right)-y^{i}(s) .
$$

\footnotetext{
${ }^{7}$ We believe that this assumption is rather innocuous given that a supplier's pseudo-type is monotonically decreasing in his type. It may well be that it follows from the other properties, but this question is still open. This property of the pseudo-type mapping is also needed in order to derive the results in Asker and Cantillon (2007). However, they do not make it an explicit assumption and also do not show, how it may follow from other properties. We consider this question also in their setting as still open.

${ }^{8}$ Note that $\tilde{x}^{i}(s) t\left(\theta^{i}\right)$ and $x^{i}(s) w^{i}(s)$ are dot products. Other instances of dot products in the remainder of the paper are not especially pointed out.
} 
The corresponding buyer utility for the outcome $(x(s), w(s))$ if suppliers have the type profile $\theta$ is

$$
\begin{aligned}
u^{0}(s \mid \theta) & =\sum_{i=1}^{n} \sum_{j=1}^{d} x_{j}^{i}(s)\left(v_{j}\left(q_{j}^{* i}\left(\theta^{i}\right)\right)-p_{j}^{i}\left(s, q_{j}^{i *}\left(\theta^{i}\right)\right)\right)+\sum_{i=1}^{n} x_{0}^{i}(s) w_{0}^{i}(s) \\
& =\sum_{i=1}^{n} \sum_{j=1}^{d} x_{j}^{i}(s)\left(v_{j}\left(q_{j}^{* i}\left(\theta^{i}\right)\right)-\phi_{j}\left(q_{j}^{* i}\left(\theta^{i}\right)\right)+w_{j}^{i}(s)\right)+\sum_{i=1}^{n} x_{0}^{i}(s) w_{0}^{i}(s) \\
& =\sum_{i=1}^{n} \tilde{x}^{i}(s)\left(v\left(q^{* i}\left(\theta^{i}\right)\right)-\phi\left(q^{* i}\left(\theta^{i}\right)\right)\right)+x^{i}(s) w^{i}(s) \\
& =\sum_{i=1}^{n} \tilde{x}^{i}(s)\left(v\left(q^{* i}\left(\theta^{i}\right)\right)-\phi\left(q^{* i}\left(\theta^{i}\right)\right)\right)+y^{i}(s) .
\end{aligned}
$$

The second equality follows from the fact that the price supplier $i$ is asking for item set $j$ is determined by his optimal quality level and the winning score that he has to satisfy (see also (3)). Specifically, $p_{j}^{i}\left(s, q_{j}^{* i}\left(\theta^{i}\right)\right)=\phi_{j}\left(q_{j}^{* i}\left(\theta^{i}\right)\right)-w_{j}^{i}(s)$. The third equality follows from defining $v\left(q^{* i}\left(\theta^{i}\right)\right)=\left(v_{1}\left(q_{1}^{* i}\left(\theta^{i}\right)\right), \ldots, v_{d}\left(q_{d}^{* i}\left(\theta^{i}\right)\right)\right)$ and a similar definition for $\phi\left(q^{* i}\left(\theta^{i}\right)\right)$. The last equality follows from (7).

Supplier $i$ 's bidding strategy $\beta^{i}$ maps each of his possible types $\theta^{i} \in \Theta$ into a price-quality bid $\left(p^{i}, q^{i}\right) \in \mathbb{R}_{+}^{d+m}$. Together with the scoring rule, $\beta^{i}$ implies a scored bidding strategy $b^{i}$ mapping each of $i$ 's types into a vector of scores $s^{i} \in \mathbb{R}^{d}$. Note that for the determination of outcomes (allocation vectors and winning scores), and hence the determination of the buyer's and suppliers' utilities, only the reported scores are of importance and not the pricequality bids that generated them. Therefore we concentrate in the following on scored bidding strategies. $^{9}$

If supplier $i$ of type $\theta^{i}$ believes that all other suppliers bid according to the profile of scored bidding strategies $b^{-i}=\left(b^{1}, \ldots, b^{i-1}, b^{i+1}, \ldots, b^{n}\right)$, his expected utility if making a scored bid $s^{i}$ is

$$
\begin{aligned}
U^{i}\left(s^{i} \mid \theta^{i}\right) & =\int_{\Theta^{-i}}\left(\tilde{x}^{i}\left(s^{i}, b^{-i}\left(\theta^{-i}\right)\right) t\left(\theta^{i}\right)-y^{i}\left(s^{i}, b^{-i}\left(\theta^{-i}\right)\right)\right) \gamma^{-i}\left(\theta^{-i}\right) d \theta^{-i} \\
& =E_{-i}\left[\tilde{x}^{i}\left(s^{i}, b^{-i}\left(\theta^{-i}\right)\right) t\left(\theta^{i}\right)-y^{i}\left(s^{i}, b^{-i}\left(\theta^{-i}\right)\right)\right] .
\end{aligned}
$$

\footnotetext{
${ }^{9}$ The analysis done in the following section goes also through (in slightly adapted form) if one allows for mixed strategies, that is, $b^{i}$ maps each of supplier $i$ 's types into a distribution over finitely many score vectors. To simplify matters we stick to pure strategies.
} 
The expected utility of the buyer if suppliers bid according to the profile of scored bidding strategies $b=\left(b^{1}, \ldots, b^{n}\right)$ is

$$
U^{0}(b)=\int_{\theta \in \Theta^{n}}\left[\sum_{i=1}^{n} \tilde{x}^{i}(b(\theta))\left(v\left(q^{* i}\left(\theta^{i}\right)\right)-\phi\left(q^{* i}\left(\theta^{i}\right)\right)\right)+y^{i}(b(\theta))\right] \gamma(\theta) d \theta .
$$

The profile of scored bidding strategies $b$ constitutes a Bayes-Nash equilibrium if for every supplier $i$ and all types $\theta^{i} \in \Theta$

$$
\begin{array}{rlr} 
& E_{-i}\left[\tilde{x}^{i}\left(b^{i}\left(\theta^{i}\right), b^{-i}\left(\theta^{-i}\right)\right) t\left(\theta^{i}\right)-y^{i}\left(b^{i}\left(\theta^{i}\right), b^{-i}\left(\theta^{-i}\right)\right)\right] \\
\geq \quad & E_{-i}\left[\tilde{x}^{i}\left(s^{i}, b^{-i}\left(\theta^{-i}\right)\right) t\left(\theta^{i}\right)-y^{i}\left(s^{i}, b^{-i}\left(\theta^{-i}\right)\right)\right] & \forall s^{i} .
\end{array}
$$

Supplier $i$ 's scored bidding strategy might assign different bids to types yielding the same pseudo-type. Given a profile of scored bidding strategies of the other suppliers, those bids either induce the same expected allocation vector for $i$ or differing expected allocation vectors. We conclude this section by formalizing this observation and introduce the following properties for suppliers' scored bidding strategies:

Definition 1 (Allocation Mixing) Consider the profile of scored bidding strategies b. Supplier $i$ 's strategy $b^{i}$ employs allocation mixing if there exist types $\theta^{i}, \hat{\theta}^{i} \in \Theta$ with $t\left(\theta^{i}\right)=t\left(\hat{\theta}^{i}\right)$ for which $b^{i}\left(\theta^{i}\right) \neq b^{i}\left(\hat{\theta}^{i}\right)$ and

$$
E_{-i}\left[\tilde{x}^{i}\left(b^{i}\left(\theta^{i}\right), b^{-i}\left(\theta^{-i}\right)\right)\right] \neq E_{-i}\left[\tilde{x}^{i}\left(b^{i}\left(\hat{\theta}^{i}\right), b^{-i}\left(\theta^{-i}\right)\right)\right] .
$$

This property deals with the expected allocation vectors induced by bids from types yielding the same pseudo-type. Suppose that there are some types yielding the same pseudo-type for which supplier $i$ makes different scored bids. Allocation mixing means that, given a strategy profile $b^{-i}$ of the others, the expected allocation vectors for $i$ induced by those bids are not the same. Furthermore, we define

Definition 2 (Allocation Equivalent Mixing) Consider the profile of scored bidding strategies b. Supplier $i$ 's strategy $b^{i}$ employs allocation equivalent mixing if there exist types $\theta^{i}, \hat{\theta}^{i} \in$ $\Theta$ with $t\left(\theta^{i}\right)=t\left(\hat{\theta}^{i}\right)$ for which $b^{i}\left(\theta^{i}\right) \neq b^{i}\left(\hat{\theta}^{i}\right)$ but

$$
E_{-i}\left[\tilde{x}^{i}\left(b^{i}\left(\theta^{i}\right), b^{-i}\left(\theta^{-i}\right)\right)\right]=E_{-i}\left[\tilde{x}^{i}\left(b^{i}\left(\hat{\theta}^{i}\right), b^{-i}\left(\theta^{-i}\right)\right)\right] .
$$


Similar to the first property this one also deals with the expected allocation vectors induced by bids from types yielding the same pseudo-type. Suppose that there are some types yielding the same pseudo-type for which supplier $i$ makes different scored bids. Allocation equivalent mixing means that, given a strategy profile $b^{-i}$ of the others, the expected allocation vectors for $i$ induced by those bids are the same.

In the next section we are going establish a link between equilibria in the scoring auction which are based on types (that is, suppliers' bidding strategies can specify different bids for different types) and the ones which are based on pseudo-types (that is, suppliers' bidding strategies specify the same bid for all types yielding the same pseudo-type). For this we finally define

Definition 3 (Almost Everywhere (A.E.) Coinciding Strategies) We say that the profile of scored bidding strategies $b$ based on types coincides with the strategy profile $\hat{b}$ based on pseudo-types a.e. if $b$ and $\hat{b}$ differ only on a set of types with Lebesque measure zero.

\section{Pseudo-types are Sufficient Statistics}

In this section we show that if every supplier's bidding strategy employs allocation equivalent mixing (see Definition 2) only for a set of types with Lebesgue measure zero, then in order to analyse the set of possible equilibria in a scoring auction and the corresponding expected utilities of the buyer it is sufficient to consider a restricted setting where suppliers bid based on their pseudo-types. That is, suppliers make the same scored bid for all types yielding the same pseudo-type. Thus, we show that pseudo-types are sufficient statistics, rendering suppliers original types redundant for the analysis of the scoring auction equilibria.

In a first step we show that every equilibrium in the scoring auction is outcome equivalent to a mixed strategy equilibrium where each supplier $i$ associates all types yielding the same pseudo-type with the same mixed bid. Two equilibria are outcome equivalent if they both induce the same distribution over outcomes (that is allocation vectors and winning scores).

Lemma 2 For every equilibrium $b$ in the scoring auction there exists an outcome equivalent mixed strategy equilibrium $\tilde{b}$ such that $\tilde{b}^{i}\left(\theta^{i}\right)=\tilde{b}^{i}\left(\hat{\theta}^{i}\right)$ whenever $t\left(\theta^{i}\right)=t\left(\hat{\theta}^{i}\right)$.

\section{Proof}

Let $b=\left(b^{1} \ldots, b^{n}\right)$ be an equilibrium in the scoring auction. For each supplier $i$ and each 
pseudo-type $t^{i} \in T$ consider the mixed bid $r^{i}\left(t^{i}\right)$ mapping $t^{i}$ into a distribution over vectors of scores. The support of $r^{i}\left(t^{i}\right)$ consists of the different scored bids generated by $b^{i}$ for all the types yielding pseudo-type $t^{i}$, that is, the support set is $\left\{b^{i}\left(\theta^{i}\right) \mid \theta^{i} \in \Theta_{t^{i}}\right\}$. Furthermore, each scored bid in the support of $r^{i}\left(t^{i}\right)$ is played with the relative frequency with which it is played by all the types in $\Theta_{t^{i}}$. Now, we can construct a new equilibrium where each supplier $i$ has the same bidding strategy for all types yielding the same pseudo-type by assigning to all $\theta^{i} \in \Theta_{t^{i}}$ the same mixed bid $r^{i}\left(t^{i}\right)$. That is, for all $\theta^{i} \in \Theta$ we define $\tilde{b}^{i}\left(\theta^{i}\right)=r^{i}\left(t\left(\theta^{i}\right)\right)$. That $\tilde{b}=\left(\tilde{b}^{1}, \ldots, \tilde{b}^{n}\right)$ is indeed an equilibrium can be seen as follows.

First, note that by construction the distribution of bids coming from each supplier $i$ remains unchanged when he switches from $b^{i}$ to $\tilde{b}^{i}$. Second, consider supplier $i$ 's expected utility. Since $b$ is an equilibrium we have by definition (see (12)) for all $\theta^{i} \in \Theta$ that

$$
b^{i}\left(\theta^{i}\right) \in \arg \max _{s^{i}} E_{-i}\left[\tilde{x}^{i}\left(s^{i}, b^{-i}\left(\theta^{-i}\right)\right) t\left(\theta^{i}\right)-y^{i}\left(s^{i}, b^{-i}\left(\theta^{-i}\right)\right)\right] .
$$

Since supplier $i$ 's private information enters his expected utility only via his pseudo-type, he is indifferent about the bids adapted by all the types yielding the same pseudo-type. That is, the bid $b^{i}\left(\theta^{i}\right)$ is also a best reply for all other $\hat{\theta}^{i} \in \Theta_{t\left(\theta^{i}\right)}$. Hence, every bid in the support of $\tilde{b}^{i}\left(\theta^{i}\right)$ is a best reply for all $\hat{\theta}^{i} \in \Theta_{t\left(\theta^{i}\right)}$. It follows that $\tilde{b}^{i}$ is a best response for supplier $i$ against $b^{-i}$. Since, as mentioned before, the distribution of bids coming from the other suppliers is not changing when switching from $b^{-i}$ to $\tilde{b}^{-i}, \tilde{b}^{i}$ is also a best response against $\tilde{b}^{-i}$. So, $\tilde{b}$ is an equilibrium. Furthermore, $b$ and $\tilde{b}$ are outcome equivalent as both equilibria induce the same distribution over scored bids and therefore also the same distribution over outcomes.

Above we have seen that the suppliers are indifferent between an equilibrium $b$ and its outcome equivalent mixed counterpart $\tilde{b}$ constructed as in the proof of Lemma 2 since their expected utilities are the same in both. The buyer is also indifferent between these two equilibria. However, this cannot as easily be observed as in the suppliers' case, and we have to make use of the following lemma in order to show it. (Asker and Cantillon (2007) illustrate this difficulty for a special case of our model where only one item is to be allocated among the suppliers.)

The next result considers the equilibrium $\tilde{b}$ constructed for Lemma 2 above where all types yielding the same pseudo-type $t^{i}$ have the same mixed bid $r^{i}\left(t^{i}\right)$. We show that for 
every supplier $i, r^{i}($.$) is not mixing over pure bids which induce different expected allocations$ for $i$, except possibly on a set of measure zero.

Lemma 3 Consider the equilibrium $\tilde{b}$ constructed in Lemma 2 where every type yielding pseudo-type $t^{i}$ has the same mixed bid $r^{i}\left(t^{i}\right)$. For every supplier $i$, the set of pseudo-types $t^{i} \in T$ for which there exist elements $s^{i}, \hat{s}^{i}$ in the support of $r^{i}\left(t^{i}\right)$ such that

$$
E_{-i}\left[\tilde{x}^{i}\left(s^{i}, \tilde{b}^{-i}\left(\theta^{-i}\right)\right)\right] \neq E_{-i}\left[\tilde{x}^{i}\left(\hat{s}^{i}, \tilde{b}^{-i}\left(\theta^{-i}\right)\right)\right]
$$

has Lebesgue measure zero. ${ }^{10}$

\section{Proof}

Suppose that $b=\left(b^{1}, \ldots, b^{n}\right)$ is an equilibrium in the scoring auction and that $\tilde{b}=\left(\tilde{b}^{1}, \ldots, \tilde{b}^{n}\right)$ is the mixed strategy equilibrium constructed as described in the proof of Lemma 2. Remember that in $\tilde{b}$ all of supplier $i$ 's types yielding the same pseudo-type $t^{i}$ have the same mixed bid $r^{i}\left(t^{i}\right)$. Take some supplier $i$ and some pseudo-type $t^{i} \in T$. Each scored bid $s^{i}$ in the support of $r^{i}\left(t^{i}\right)$ yields an expected allocation vector $E_{-i}\left[x^{i}\left(s^{i}, \tilde{b}^{-i}\left(\theta^{-i}\right)\right)\right] \in[0,1]^{2^{a}}$. The first element of this expected allocation vector, consisting of $i$ 's expected probability to win item set 1 , is denoted by $E_{-i}\left[x^{i}\left(s^{i}, \tilde{b}^{-i}\left(\theta^{-i}\right)\right)\right]_{1}$. Let $\underline{s}^{i}$ and $\bar{s}^{i}$ denote the elements in the support of $r^{i}\left(t^{i}\right)$ for which

$$
E_{-i}\left[x^{i}\left(\underline{s}^{i}, \tilde{b}^{-i}\left(\theta^{-i}\right)\right)\right]_{1} \leq E_{-i}\left[x^{i}\left(s^{i}, \tilde{b}^{-i}\left(\theta^{-i}\right)\right)\right]_{1}
$$

and

$$
E_{-i}\left[x^{i}\left(\bar{s}^{i}, \tilde{b}^{-i}\left(\theta^{-i}\right)\right)\right]_{1} \geq E_{-i}\left[x^{i}\left(s^{i}, \tilde{b}^{-i}\left(\theta^{-i}\right)\right)\right]_{1}
$$

for all $s^{i}$ in the support of $r^{i}\left(t^{i}\right)$. Thus, considering all the elements in the support of $r^{i}\left(t^{i}\right)$, $\underline{s}^{i}$ is yielding the lowest expected probability for supplier $i$ to win item set 1 , whereas $\bar{s}^{i}$ is yielding the highest expected winning probability. ${ }^{11}$ Let $t_{*}^{i} \in T$ be a pseudo-type that differs from $t^{i}$ only in its first element, that is $t_{* 1}^{i} \neq t_{1}^{i}$ but $t_{* h}^{i}=t_{h}^{i} \forall h \in\{2, \ldots, d\}$. In the same way as above we define $\underline{s}_{*}^{i}$ and $\bar{s}_{*}^{i}$.

\footnotetext{
${ }^{10} E_{-i}\left[\tilde{x}^{i}\left(s^{i}, \tilde{b}^{-i}\left(\theta^{-i}\right)\right)\right]$ is the expected allocation vector for supplier $i$ if he makes a scored bid $s^{i}$ and the other suppliers bid according to $\tilde{b}^{-i}$. Given that $\tilde{b}^{-i}$ is a profile of mixed bidding strategies, note that in this case $E_{-i}[$.$] denotes the expectation taken over all type profiles \theta^{-i}$ as well as the corresponding mixed strategies $\tilde{b}^{-i}\left(\theta^{-i}\right)$.

${ }^{11}$ If $r^{i}\left(t^{i}\right)$ is not mixing then by construction $\underline{s}^{i}=\bar{s}^{i}$ and consequently $E_{-i}\left[x^{i}\left(\underline{s}^{i}, \tilde{b}^{-i}\left(\theta^{-i}\right)\right)\right]=$ $E_{-i}\left[x^{i}\left(\bar{s}^{i}, \tilde{b}^{-i}\left(\theta^{-i}\right)\right)\right]$.
} 
From $\tilde{b}$ being an equilibrium and every element in the support of $r^{i}\left(t^{i}\right)$ being a best response against $\tilde{b}^{-i}$ for all types $\theta^{i} \in \Theta_{t^{i}}$ (see also explanation in the proof of Lemma 2) it follows that

$$
\begin{aligned}
& E_{-i}\left[x^{i}\left(\underline{s}^{i}, \tilde{b}^{-i}\left(\theta^{-i}\right)\right) t^{i}-y^{i}\left(\underline{s}^{i}, \tilde{b}^{-i}\left(\theta^{-i}\right)\right)\right] \\
\geq & E_{-i}\left[x^{i}\left(\underline{s}_{*}^{i}, \tilde{b}^{-i}\left(\theta^{-i}\right)\right) t^{i}-y^{i}\left(\underline{s}_{*}^{i}, \tilde{b}^{-i}\left(\theta^{-i}\right)\right)\right]
\end{aligned}
$$

and

$$
\begin{aligned}
& E_{-i}\left[x^{i}\left(\underline{s}_{*}^{i}, \tilde{b}^{-i}\left(\theta^{-i}\right)\right) t_{*}^{i}-y^{i}\left(\underline{s}_{*}^{i}, \tilde{b}^{-i}\left(\theta^{-i}\right)\right)\right] \\
\geq & E_{-i}\left[x^{i}\left(\underline{s}^{i}, \tilde{b}^{-i}\left(\theta^{-i}\right)\right) t_{*}^{i}-y^{i}\left(\underline{s}^{i}, \tilde{b}^{-i}\left(\theta^{-i}\right)\right)\right] .
\end{aligned}
$$

Adding (13) and (14) yields

$$
\begin{aligned}
& E_{-i}\left[x^{i}\left(\underline{s}^{i}, \tilde{b}^{-i}\left(\theta^{-i}\right)\right)\right] t^{i}+E_{-i}\left[x^{i}\left(\underline{s}_{*}^{i}, \tilde{b}^{-i}\left(\theta^{-i}\right)\right)\right] t_{*}^{i} \\
\geq \quad & E_{-i}\left[x^{i}\left(\underline{s}_{*}^{i}, \tilde{b}^{-i}\left(\theta^{-i}\right)\right)\right] t^{i}+E_{-i}\left[x^{i}\left(\underline{s}^{i}, \tilde{b}^{-i}\left(\theta^{-i}\right)\right)\right] t_{*}^{i} .
\end{aligned}
$$

By rearranging the terms in the above inequality we get

$$
\left(E_{-i}\left[x^{i}\left(\underline{s}^{i}, \tilde{b}^{-i}\left(\theta^{-i}\right)\right)\right]-E_{-i}\left[x^{i}\left(\underline{s}_{*}^{i}, \tilde{b}^{-i}\left(\theta^{-i}\right)\right)\right]\right)\left(t^{i}-t_{*}^{i}\right) \geq 0
$$

Since only $t_{* 1}^{i} \neq t_{1}^{i}$, whereas all the other elements of the two pseudo-types are the same, this inequality becomes

$$
\left(E_{-i}\left[x^{i}\left(\underline{s}^{i}, \tilde{b}^{-i}\left(\theta^{-i}\right)\right)\right]_{1}-E_{-i}\left[x^{i}\left(\underline{s}_{*}^{i}, \tilde{b}^{-i}\left(\theta^{-i}\right)\right)\right]_{1}\right)\left(t_{1}^{i}-t_{* 1}^{i}\right) \geq 0 .
$$

Thus, $E_{-i}\left[x^{i}\left(\underline{s}^{i}, \tilde{b}^{-i}\left(\theta^{-i}\right)\right)\right]_{1}$ is monotonically increasing in $t_{1}^{i}$. This implies that $E_{-i}\left[x^{i}\left(\underline{s}^{i}, \tilde{b}^{-i}\left(\theta^{-i}\right)\right)\right]_{1}$ is Riemann integrable (see for example Khuri (2003), Theorem 6.3.2) and hence continuous a.e. (see for example Wrede and Spiegel (2002), p.91). By performing the same steps as above for $\bar{s}^{i}$ and $\bar{s}_{*}^{i}$ we find that also $E_{-i}\left[x^{i}\left(\bar{s}^{i}, \tilde{b}^{-i}\left(\theta^{-i}\right)\right)\right]_{1}$ is continuous a.e..

Similar to the above, from $\tilde{b}$ being an equilibrium and every element in the support of $r^{i}\left(t^{i}\right)$ being a best response against $\tilde{b}^{-i}$ for all types $\theta^{i} \in \Theta_{t^{i}}$ it also follows that

$$
\begin{aligned}
& E_{-i}\left[x^{i}\left(\underline{s}^{i}, \tilde{b}^{-i}\left(\theta^{-i}\right)\right) t^{i}-y^{i}\left(\underline{s}^{i}, \tilde{b}^{-i}\left(\theta^{-i}\right)\right)\right] \\
\geq \quad & E_{-i}\left[x^{i}\left(\bar{s}_{*}^{i}, \tilde{b}^{-i}\left(\theta^{-i}\right)\right) t^{i}-y^{i}\left(\bar{s}_{*}^{i}, \tilde{b}^{-i}\left(\theta^{-i}\right)\right)\right]
\end{aligned}
$$


and

$$
\begin{aligned}
& E_{-i}\left[x^{i}\left(\bar{s}_{*}^{i}, \tilde{b}^{-i}\left(\theta^{-i}\right)\right) t_{*}^{i}-y^{i}\left(\bar{s}_{*}^{i}, \tilde{b}^{-i}\left(\theta^{-i}\right)\right)\right] \\
\geq \quad & E_{-i}\left[x^{i}\left(\underline{s}^{i}, \tilde{b}^{-i}\left(\theta^{-i}\right)\right) t_{*}^{i}-y^{i}\left(\underline{s}^{i}, \tilde{b}^{-i}\left(\theta^{-i}\right)\right)\right] .
\end{aligned}
$$

Performing the same steps as above, (15) and (16) imply that

$$
\left(E_{-i}\left[x^{i}\left(\underline{s}^{i}, \tilde{b}^{-i}\left(\theta^{-i}\right)\right)\right]_{1}-E_{-i}\left[x^{i}\left(\bar{s}_{*}^{i}, \tilde{b}^{-i}\left(\theta^{-i}\right)\right)\right]_{1}\right)\left(t_{1}^{i}-t_{* 1}^{i}\right) \geq 0 .
$$

Thus, if $t_{1}^{i}>t_{* 1}^{i}$ then

$$
E_{-i}\left[x^{i}\left(\underline{s}^{i}, \tilde{b}^{-i}\left(\theta^{-i}\right)\right)\right]_{1} \geq E_{-i}\left[x^{i}\left(\bar{s}_{*}^{i}, \tilde{b}^{-i}\left(\theta^{-i}\right)\right)\right]_{1} .
$$

Since $E_{-i}\left[x^{i}\left(\bar{s}^{i}, \tilde{b}^{-i}\left(\theta^{-i}\right)\right)\right]_{1}$ is continuous a.e. we have that

$$
E_{-i}\left[x^{i}\left(\bar{s}_{*}^{i}, \tilde{b}^{-i}\left(\theta^{-i}\right)\right)\right]_{1} \rightarrow E_{-i}\left[x^{i}\left(\bar{s}^{i}, \tilde{b}^{-i}\left(\theta^{-i}\right)\right)\right]_{1} \quad \text { a.e. }
$$

as $t_{* 1}^{i} \rightarrow t_{1}^{i}$. It follows that

$$
E_{-i}\left[x^{i}\left(\underline{s}^{i}, \tilde{b}^{-i}\left(\theta^{-i}\right)\right)\right]_{1} \geq E_{-i}\left[x^{i}\left(\bar{s}^{i}, \tilde{b}^{-i}\left(\theta^{-i}\right)\right)\right]_{1} \quad \text { a.e. }
$$

Since by definition of $\underline{s}^{i}$ and $\bar{s}^{i}$

$$
E_{-i}\left[x^{i}\left(\bar{s}^{i}, \tilde{b}^{-i}\left(\theta^{-i}\right)\right)\right]_{1} \geq E_{-i}\left[x^{i}\left(\underline{s}^{i}, \tilde{b}^{-i}\left(\theta^{-i}\right)\right)\right]_{1}
$$

this implies that

$$
E_{-i}\left[x^{i}\left(\underline{s}^{i}, \tilde{b}^{-i}\left(\theta^{-i}\right)\right)\right]_{1}=E_{-i}\left[x^{i}\left(\bar{s}^{i}, \tilde{b}^{-i}\left(\theta^{-i}\right)\right)\right]_{1} \quad \text { a.e. }
$$

By repeating the procedure described above for the remaining $d-1$ elements of the pseudotype we find that almost everywhere it holds, that for all scored bids $s^{i}, \hat{s}^{i}$ in the support of $r^{i}\left(t^{i}\right)$

$$
E_{-i}\left[x^{i}\left(s^{i}, \tilde{b}^{-i}\left(\theta^{-i}\right)\right)\right]=E_{-i}\left[x^{i}\left(\hat{s}^{i}, \tilde{b}^{-i}\left(\theta^{-i}\right)\right)\right] .
$$

That is, the set of pseudo-types $t^{i}$ for which there exist elements $s^{i}, \hat{s}^{i}$ in the support of $r^{i}\left(t^{i}\right)$ such that $E_{-i}\left[\tilde{x}^{i}\left(s^{i}, \tilde{b}^{-i}\left(\theta^{-i}\right)\right)\right] \neq E_{-i}\left[\tilde{x}^{i}\left(\hat{s}^{i}, \tilde{b}^{-i}\left(\theta^{-i}\right)\right)\right]$ has Lebesgue measure zero.

Before we have already seen that, because their expected utilities are same in both, the suppliers are indifferent between an equilibrium $b$ and its outcome equivalent counterpart $\tilde{b}$, 
constructed as in the proof of Lemma 2. Using the above Lemma 3 we can now claim the same for the buyer. The details of the proof are stated in the appendix.

Remember that the support of the mixed bid $r^{i}\left(t^{i}\right)$, used in the construction of supplier $i$ 's new equilibrium strategy $\tilde{b}^{i}$ in the proof of Lemma 2, consists of the different scored bids generated by the original equilibrium strategy $b^{i}$ for all the types in $\Theta_{t^{i}}$. Suppose that for some pseudo-type $t^{i}$ there exist scored bids in the support of $r^{i}\left(t^{i}\right)$ which yield different expected allocation vectors for $i$. Then, this means that $b^{i}$ employs allocation mixing (see Definition 1 in the foregoing section) for the types in $\Theta_{t^{i}}$. Similarly, if such scored bids do not exist in the support of $r^{i}\left(t^{i}\right)$ then $b^{i}$ does not employ allocation mixing for the types in $\Theta_{t^{i}}$. Based on this observation we find the following result:

Lemma 4 In every equilibrium $b$ in the scoring auction, supplier $i$ 's bidding strategy $b^{i}$ employs allocation mixing only for a set of types $\theta^{i} \in \Theta$ that has Lebesgue measure zero.

\section{Proof}

Suppose that $b$ is an equilibrium in the scoring auction. Take some pseudo-type $t^{i} \in T$ and consider the types yielding this pseudo-type. If there exist $\theta^{i}, \hat{\theta}^{i} \in \Theta_{t^{i}}$ such that $E_{-i}\left[\tilde{x}^{i}\left(b^{i}\left(\theta^{i}\right), b^{-i}\left(\theta^{-i}\right)\right)\right] \neq E_{-i}\left[\tilde{x}^{i}\left(b^{i}\left(\hat{\theta}^{i}\right), b^{-i}\left(\theta^{-i}\right)\right)\right]$ then $b^{i}$ employs allocation mixing for $\Theta_{t^{i}}^{i}$. From Lemma 3 it follows that the set of pseudo-types for which this happens has Lebesgue measure zero. This implies that also the corresponding set of types yielding these pseudo-types has Lebesgue measure zero. (Remember from the foregoing section that one property of the pseudo-type mapping is: If a set of pseudo-types has Lebesgue measure zero then the set of types yielding this set of pseudo-types has also Lebesgue measure zero.)

Now, restricting our attention to the class of equilibria in the scoring auction where suppliers employ allocation equivalent mixing only for a zero measure of types, we can state based on Lemma 4 the following main result of this section:

Theorem 1 Suppose that the set of types for which suppliers employ allocation equivalent mixing in their bidding strategies has Lebesgue measure zero. Then, for every equilibrium in the scoring auction based on types there exists an equilibrium based on pseudo-types that coincides with it a.e. (see Definition 3). Vice versa, every equilibrium in the restricted setting, where strategies are based on pseudo-types, is also an equilibrium in the unrestricted setting. 


\section{Proof}

Trivially, all equilibria in the scoring auction where suppliers are constrained to make the same bid for all types yielding the same pseudo-type are also equilibria in the unconstrained scoring auction.

For the opposite direction, suppose that suppliers employ allocation equivalent mixing in their biding strategies only for a zero measure of types and that $b$ is an equilibrium in the unconstrained scoring auction. Based on $b$ we can construct an equilibrium $\hat{b}$ in which suppliers make the same bid for types yielding the same pseudo-type and which differs from $b$ at most on a set of measure zero. This is done as follows: For every supplier $i$ and every $t^{i} \in T$ pick some $\bar{\theta}^{i} \in \Theta_{t^{i}}$ and set $\hat{b}^{i}\left(\theta^{i}\right)=b^{i}\left(\bar{\theta}^{i}\right), \forall \theta^{i} \in \Theta_{t^{i}}$. Since $i$ 's private information enters his expected utility only via his pseudo-type, $\hat{b}^{i}$ is also best response against $b^{-i}$ (see argument in the proof of Lemma 2). From Lemma 4 it follows that for every supplier $i, \hat{b}^{i}$ differs from $b^{i}$ only for a set of types $\theta^{i} \in \Theta$ that has Lebesgue measure zero. Hence, $\hat{b}^{i}$ is also a best response against $\hat{b}^{-i}$.

Considering the proof above, note that supplier $i$ switching from his original, unconstrained scored bidding strategy $b^{i}$ to the constrained one, $\hat{b}^{i}$, is inconsequential to his own expected utility because his private information enters it only via the pseudo-type (again, see argument in the proof of Lemma 2). However, if $\hat{b}^{i}$ differs from $b^{i}$ on a non-zero measure of types then we cannot guarantee that for the other suppliers the strategy profile $b^{-i}$ (and hence also $\hat{b}^{-i}$ ) is still a best reply. ${ }^{12}$ We can ensure that $\hat{b}^{i}$ differs from $b^{i}$ only on a zero measure of types if $b^{i}$ employs allocation mixing and allocation equivalent mixing only for a

\footnotetext{
${ }^{12}$ In order to illustrate this point consider the following simple game. There are only two players and each player has two possible actions - player 1 has action set $\{U, D\}$, and player 2's action set is $\{L, R\}$. The resulting utilities for both players are as follows:

\begin{tabular}{c|c|c|}
\multicolumn{1}{c}{$L$} & \multicolumn{1}{c}{$R$} \\
\cline { 2 - 3 }$U$ & 0,2 & 0,1 \\
\cline { 2 - 3 }$D$ & 0,0 & 0,1 \\
\cline { 2 - 3 } & &
\end{tabular}

Notice that, no matter what player 2 does, player 1's utility is always 0 . One can easily see that for example player 1 playing $U$ with probability $\frac{1}{3}$ and $D$ with probability $\frac{2}{3}$ and player 2 playing $R$ is an equilibrium. If player 1 switches to playing $U$ with probability 1 , this is inconsequential to his own expected utility. However, player 2 could now increase his expected utility by switching from playing $R$ to playing $L$.
} 
zero measure of types. With respect to allocation mixing we can verify this analytically (see Lemma 4). However, for allocation equivalent mixing we cannot do this and have to exclude it by assumption.

Thus, if allocation equivalent mixing is essentially absent, we can confine ourselves for the analysis of the set of possible equilibria in the scoring auction to a restricted setting where suppliers bid only based on their pseudo-types. Note that the expected utilities of the buyer and the suppliers are the same in a type-based equilibrium and in the corresponding pseudotype based equilibrium that coincides with it almost everywhere (constructed as above in the proof of Theorem 1).

Concerning the link between Theorem 1 and the main result of Asker and Cantillon (2007, Theorem 1) with regard to the sufficiency of pseudo-types for the analysis of single item scoring auctions, we observe the following. Suppliers' equilibrium bidding strategies, mapping their possible types into vectors of scores, together with the allocation rule and the winning score rule imply an equilibrium outcome function, mapping suppliers' possible types into outcomes $(x(),. y()$.$) . From Theorem 1$ it readily follows that, again if suppliers essentially do not employ allocation equivalent mixing, for every equilibrium in the scoring auction based on types there exists an equilibrium based on pseudo-types that induces the same equilibrium outcome function, except possibly on a set of types with Lebesgue measure zero. This finding is the direct combinatorial scoring auction extension of Asker and Cantillon's (2007) main result about the role of pseudo-types as sufficient statistics in single item scoring auctions (their Theorem 1) which is stated in terms of equilibrium outcome functions rather than the underlying equilibrium bidding strategies (like our Theorem 1).

\section{Application}

The main result of the foregoing section is the multi-item extension of Asker and Cantillon's (2007) result that pseudo-types are a sufficient statistic in quasi-linear single item scoring auctions. They use their result to establish a link to the well studied standard single item IPV (independent private values) auctions and demonstrate how knowledge about these auctions can be used for the equilibrium analysis in single item scoring auctions. Similar to them, we can establish a link between combinatorial scoring auctions and the regular price-only combinatorial IPV auctions. 
Observe that for every combinatorial scoring auction described in Section 2 there exists a related combinatorial price-only auction allocating $a$ items to $n$ bidders where

- the allocation rule is $x$,

- the payment rule is $y=\left(y^{1}, \ldots, y^{n}\right)$ (see (7) for definition),

- bidder $i$ 's type is his pseudo-type and is distributed accordingly,

- bidder $i$ 's utility function is specified according to (8), that is given a true type $t^{i}$ his utility for a profile of reports $s=\left(s^{1}, \ldots, s^{n}\right)$ is

$$
u^{i}\left(s \mid t^{i}\right)=\tilde{x}^{i}(s) t^{i}-y^{i}(s)
$$

(see right above (6) for the definition of $\tilde{x}^{i}$ ).

Note that a bidding strategy in the above price-only auction is a mapping $T \mapsto \mathbb{R}^{d}$. Every bidding strategy like that can be seen as a pseudo-type based bidding strategy $\Theta \mapsto \mathbb{R}^{d}$ that specifies the same bid for all types yielding the same pseudo-type. Based on the above observations and Theorem 1 we can state the following:

Corollary 1 Suppose that the set of types for which suppliers employ allocation equivalent mixing in their bidding strategies has Lebesgue measure zero. Then, for every equilibrium in the scoring auction based on types there exists an equilibrium based on pseudo-types in the corresponding combinatorial price-only auction described above that coincides with it a.e. (see Definition 3). Vice versa, every equilibrium in the combinatorial price-only auction, where strategies are based on pseudo-types, is also an equilibrium in the associated scoring auction. This result is the multi-item extension of Asker and Cantillon's (2007) Corollary 1. It implies that if allocation equivalent mixing is essentially absent then we can use the existing literature on combinatorial price-only auctions to analyze the equilibria in the scoring auction. In the following we illustrate this by constructing an efficient scoring auction based on the probably most famous combinatorial auction, the Vickrey-Clarke-Groves (VCG) mechanism (Vickrey (1961), Clarke (1971), Groves (1973)). However, note that the classic combinatorial auctions literature has to offer much more than just the standard VCG auction (see Cramton, Shoham and Steinberg (2006) for an overview). A recent stream of literature for example is dealing with sufficient conditions for the existence of equilibria, see e.g. Saks and Yu (2005) 
for dominant strategy implementation and Müller, Perea and Wolf (2007) for Bayes-Nash implementation. Another interesting stream of literature is concerned with ways to reduce the communication complexity and the computational complexity of the allocation algorithm in combinatorial auctions, see e.g. Holzman, Kfir-Dahav, Monderer and Tennenholtz (2004), Nisan and Ronen (2000), Bartal, Gonen and Nisan (2003), Dobzinski, Nisan, Shapira (2006).

An additional example of auction literature that can be used for the equilibrium analysis of combinatorial scoring auctions is the work of Krishna and Maenner (2001) on the bidder utility in direct revelation mechanisms with multi-dimensional private information. Their results imply the following extension of Asker and Cantillon's (2007) "Expected Utility Equivalence"Theorem for single item scoring auctions to combinatorial scoring auctions. Note that in the following we consider pseudo-type based equilibria where a supplier assigns the same scored bid to all types yielding the same pseudo-type. We are going to denote a supplier's bidding strategy as $b^{i}\left(t^{i}\right)$, that is directly as a mapping $T \mapsto \mathbb{R}^{d}$.

Corollary 2 Consider two scoring auctions $\left(S_{1}, \ldots, S_{d}, x, w\right)$ and $\left(S_{1}^{\prime}, \ldots, S_{d}^{\prime}, x^{\prime}, w^{\prime}\right)$ as well as a pair of corresponding pseudo-type based equilibria $b$ and $b^{\prime}$. If

$$
\begin{aligned}
& \text { 1) } S_{j}=S_{j}^{\prime}, \forall j \in\{1, \ldots, d\} \text {, } \\
& \text { 2) } x(b(t))=x^{\prime}\left(b^{\prime}(t)\right), \forall t \in T^{n} \text { and } \\
& \text { 3) } U^{i}\left(b^{i}\left(t_{*}^{i}\right) \mid t^{i}\right)=U^{\prime i}\left(b^{\prime i}\left(t_{*}^{i}\right) \mid t^{i}\right) \text { for some fixed } t_{*}^{i} \in T, \forall i \in N \text {, }
\end{aligned}
$$

then the equilibria $b$ and $b^{\prime}$ of the two auctions generate the same expected utility for the buyer.

Condition 1 states that both scoring auctions employ the same scoring rules. Condition 2 asserts that both allocation rules and equilibria yield the same allocation vectors for each possible pseudo-type realization of the suppliers. Finally, Condition 3 states that both equilibria generate the same expected utility for supplier $i$ with fixed pseudo-type $t_{*}^{i}$. In order to see why Corollary 2 holds, it is useful to observe that the buyer's expected utility (see also (11)) can be rewritten as

$$
\begin{aligned}
U^{0}(b)= & \int_{t \in T^{n}}\left[\sum_{i=1}^{n} \tilde{x}^{i}(b(t)) E_{\theta^{i} \in \Theta_{t^{i}}}\left[v\left(q^{* i}\left(\theta^{i}\right)\right)-c\left(q^{* i}\left(\theta^{i}\right), \theta^{i}\right)\right]\right] g(t) d t \\
& -\sum_{i=1}^{n} \int_{t^{i} \in T} U^{i}\left(b^{i}\left(t^{i}\right) \mid t^{i}\right) g^{i}\left(t^{i}\right) d t^{i},
\end{aligned}
$$

where $g^{i}$ denotes supplier $i$ 's density on $T, g$ denotes the joint density on $T^{n}$ and $c\left(q^{* i}\left(\theta^{i}\right), \theta^{i}\right)=$ $\left(c_{1}\left(q_{1}^{* i}\left(\theta^{i}\right), \theta^{i}\right), \ldots, c_{d}\left(q_{d}^{* i}\left(\theta^{i}\right), \theta^{i}\right)\right)$. Since the optimal quality levels chosen by a supplier 
$\left(q^{i *}\right)$ depend only on his type and the scoring rules, it follows from Conditions 1 and 2 that the first part of (17) is the same for both equilibria. Given Conditions 2 and 3, Proposition 1 of Krishna and Maenner (2001) implies that $U^{i}\left(b^{i}\left(t^{i}\right) \mid t^{i}\right)$ is the same for both equilibria and hence the second part of (17) is the same for both equilibria as well.

\subsection{The VCG Scoring Auction}

The VCG scoring auction is specified by the scoring rules $\left(S_{1}^{*}, \ldots, S_{d}^{*}\right)$, the allocation rule $x^{*}$ and the winning score rule $w^{*}$ which we define as follows. First of all, the scoring rules (see (2)) reflect the buyer's valuation, that is for all $j \in\{1, \ldots, d\}$,

$$
S_{j}^{*}\left(p_{j}, q_{j}\right)=v_{j}\left(q_{j}\right)-p_{j}
$$

Note that in this case the $j$ th element of a supplier's pseudo-type is the maximum level of welfare that this supplier can create by producing and subsequently selling the set of item $j$. The welfare generated by a profile of allocation vectors $\chi$ if suppliers have the pseudo-type profile $t$ is

$$
W(\chi, t)=\sum_{i=1}^{n} \tilde{\chi}^{i} t^{i}
$$

where $\tilde{\chi}^{i}$ denotes supplier $i$ 's allocation vector $\chi^{i}$ without the element for the empty item set (see also right above (6) for definition). ${ }^{13}$

Based on a reported profile of scores $s$ the allocation rule in the VCG scoring auction distributes items over suppliers in such a way that welfare is maximized. This yields for every supplier one set of items $j^{*}$ (possibly the empty set) that he is contracted to deliver. So, in his corresponding allocation vector $x^{* i}(s)$ we have that $x_{j^{*}}^{* i}(s)=1$ whereas $x_{j}^{* i}(s)=0$ for all $j \neq j^{*}$. Furthermore, $x^{*}(s)$ maximizes $W(., s)$. So, denoting the maximal level of welfare

\footnotetext{
${ }^{13}$ To see this, note that if $\phi_{j}()=.v_{j}(.) \forall j$ then the buyer utility in (9) reduces to

$$
u^{0}(s \mid \theta)=\sum_{i=1}^{n} y^{i}(s) .
$$
}

Adding the suppliers' utilities (given in 8) now yields

$$
u^{0}(s \mid \theta)+\sum_{i=1}^{n} u^{i}\left(s \mid \theta^{i}\right)=\sum_{i=1}^{n} \tilde{x}^{i}(s) t\left(\theta^{i}\right),
$$

so welfare depends only on the suppliers' allocation vectors and pseudo-types. 
achievable based on score profile $s$ by $W_{\max }(s)$, we have that

$$
W_{\text {max }}(s)=\sum_{i=1}^{n} \tilde{x}^{* i}(s) s^{i} .
$$

The part of this generated by other suppliers than $i$ is

$$
W_{\max }^{-i}(s)=\sum_{h \neq i} \tilde{x}^{* h}(s) s^{h}
$$

Similarly to the above we define $\widehat{W}_{\max }\left(s^{-i}\right)$ as the maximum level of welfare that can be achieved by the others without supplier $i$ being present.

The winning score rule $w^{*}$ is now defined as follows. For the item set that he is winning $\left(j^{*}\right)$, every supplier is contracted a score reflecting the marginal impact that his presence has on the welfare generated by all the others. For all other item sets the assigned score is zero. That is, given a report profile $s$,

$$
w_{j^{*}}^{* i}(s)=\widehat{W}_{\max }\left(s^{-i}\right)-W_{\max }^{-i}(s)
$$

whereas $w_{j}^{* i}(s)=0$ for all $j \neq j^{*}$. Using (7) this implies that

$$
\begin{aligned}
y^{* i}(s) & =x^{* i}(s) w^{* i}(s) \\
& =\widehat{W}_{\max }\left(s^{-i}\right)-W_{\text {max }}^{-i}(s) .
\end{aligned}
$$

In the VCG scoring auction described above, it is a dominant strategy for suppliers to make a scored bid that corresponds to their pseudo-type. That is, $b=\left(b^{1}, \ldots, b^{n}\right)$ with $b^{i}\left(\theta^{i}\right)=t\left(\theta^{i}\right)$ is a dominant strategy equilibrium in the VCG scoring auction. This result can be obtained directly by observing that the combinatorial price-only auction that relates to the VCG scoring auction above as described in the beginning of Section 4 is the renowned VCG mechanism which efficiently allocates the items and charges bidder $i$ with a payment $y^{i}$ that corresponds to the impact that his presence in the auction has on the welfare generated by the others. It is a well-known result (see for example Ausubel and Milgrom (2006)) that in the VCG mechanism it is a dominant strategy for bidders to truthfully bid their type $t^{i}$.

For completeness we state again the rationale for this result: Suppose that the other bidders have a fixed report $s^{-i}$. Then, the utility of bidder $i$ of type $t^{i}$ for making a report $s^{i}$ 
is

$$
\begin{aligned}
u^{i}\left(s \mid t^{i}\right) & =\tilde{x}^{* i}(s) t^{i}-y^{* i}(s) \\
& =\tilde{x}^{* i}(s) t^{i}+W_{\max }^{-i}(s)-\widehat{W}_{\max }\left(s^{-i}\right) \\
& \leq W_{\max }\left(t^{i}, s^{-i}\right)-\widehat{W}_{\max }\left(s^{-i}\right) \\
& =\tilde{x}^{* i}\left(t^{i}, s^{-i}\right) t^{i}+W_{\max }^{-i}\left(t^{i}, s^{-i}\right)-\widehat{W}_{\max }\left(s^{-i}\right) \\
& =\tilde{x}^{* i}\left(t^{i}, s^{-i}\right) t^{i}-y^{* i}\left(t^{i}, s^{-i}\right) \\
& =u^{i}\left(t^{i}, s^{-i} \mid t^{i}\right),
\end{aligned}
$$

where the first and fifth equality follow from (8), the second and fourth equality follow from the definition of $y^{* i}$ and the third equality follows from the definition of $x^{*}$. Thus, truthful reporting is indeed optimal.

However, note that the close link to the VCG mechanism does not only imply that the VCG scoring auction shares the same merits, like for example the implementation in dominant startegies (so a supplier does not need to know the cost functions of the other bidders in order to play his dominant strategy). It also implies that the VCG scoring auction exhibits the same weaknesses, like the communication and computational complexity. A detailed discussion of the pros and cons of the VCG mechanism can be found in Ausubel and Milgrom (2006).

\section{Acknowledgements}

The authors thank the audience of the Second Spain Italy Netherlands Meeting on Game Theory (2006) for an interesting discussion. We are especially grateful to Estelle Cantillon for a helpful conversation and her useful comments.

\section{Appendix}

Let $b=\left(b^{1}, \ldots, b^{n}\right)$ be an equilibrium in the scoring auction. In Lemma 2 we have constructed a new mixed strategy equilibrium $\tilde{b}$ which is outcome equivalent to $b$. That is, $\tilde{b}$ induces the same distribution over scored bids as $b$ and hence also the same distribution over outcomes. As pointed out before, it can be easily observed that suppliers are indifferent between $b$ and $\tilde{b}$ as their expected utilities are the same in both. The same can be claimed for the buyer, 
however the argument is not as straightforward as in the suppliers' case. The reason is that for the buyer's expected utility not only the overall distribution of outcomes is of interest, but also the question which outcomes are associated with which type-realizations of the suppliers. To illustrate this point we start by considering a simplified setting where we only change the bidding strategy of one supplier.

Consider $\hat{b}=\left(\hat{b}^{1}, b^{2}, \ldots, b^{n}\right)$ where $\hat{b}^{1}$ is constructed based on $b^{1}$ as described in the proof to Lemma 2. By the logic described in this proof, $\hat{b}$ is an outcome equivalent equilibrium to $b$. The expected utility of the buyer (given in (11)) if suppliers bid according to $b$ can be decomposed into two terms. Specifically,

$$
\begin{aligned}
U^{0}(b)= & \int_{\theta \in \Theta^{n}}\left[\sum_{i=1}^{n} \tilde{x}^{i}(b(\theta))\left(v\left(q^{* i}\left(\theta^{i}\right)\right)-\phi\left(q^{* i}\left(\theta^{i}\right)\right)\right)+y^{i}(b(\theta))\right] \gamma(\theta) d \theta \\
= & \int_{\theta \in \Theta^{n}}\left[\sum_{i=2}^{n} \tilde{x}^{i}(b(\theta))\left(v\left(q^{* i}\left(\theta^{i}\right)\right)-\phi\left(q^{* i}\left(\theta^{i}\right)\right)\right)+y^{i}(b(\theta))\right] \gamma(\theta) d \theta \\
& +\int_{\theta \in \Theta^{n}}\left[\tilde{x}^{1}(b(\theta))\left(v\left(q^{* 1}\left(\theta^{1}\right)\right)-\phi\left(q^{* 1}\left(\theta^{1}\right)\right)\right)+y^{1}(b(\theta))\right] \gamma(\theta) d \theta .
\end{aligned}
$$

Let us consider the first term in (18). Take some $i \in\{2, \ldots, n\}$ and look at

$$
\begin{aligned}
& \int_{\theta \in \Theta^{n}}\left[\tilde{x}^{i}(b(\theta))\left(v\left(q^{* i}\left(\theta^{i}\right)\right)-\phi\left(q^{* i}\left(\theta^{i}\right)\right)\right)+y^{i}(b(\theta))\right] \gamma(\theta) d \theta \\
= & \int_{\theta^{i} \in \Theta^{i}}\left[E_{-i}\left[\tilde{x}^{i}\left(b^{i}\left(\theta^{i}\right), b^{-i}\left(\theta^{-i}\right)\right)\right]\left(v\left(q^{* i}\left(\theta^{i}\right)\right)-\phi\left(q^{* i}\left(\theta^{i}\right)\right)\right)\right] \gamma^{i}\left(\theta^{i}\right) d \theta^{i} \\
& +\int_{\theta \in \Theta^{n}}\left[y^{i}(b(\theta))\right] \gamma(\theta) d \theta .
\end{aligned}
$$

Since by construction the distribution of suppliers' bids under $b$ is the same as under $\hat{b}$, the second term of (19) is not changing if we switch from equilibrium $b$ to $\hat{b}$, that is ${ }^{14}$

$$
\int_{\theta \in \Theta^{n}}\left[y^{i}(b(\theta))\right] \gamma(\theta) d \theta=\int_{\theta \in \Theta^{n}}\left[y^{i}(\hat{b}(\theta))\right] \gamma(\theta) d \theta .
$$

Similarly, since by construction $\hat{b}^{i}=b^{i}$ and the distribution of bids coming from the other suppliers is the same under $b$ and $\hat{b}$ we find that for all $\theta^{i} \in \Theta$

$$
E_{-i}\left[\tilde{x}^{i}\left(b^{i}\left(\theta^{i}\right), b^{-i}\left(\theta^{-i}\right)\right)\right]=E_{-i}\left[\tilde{x}^{i}\left(\hat{b}^{i}\left(\theta^{i}\right), \hat{b}^{-i}\left(\theta^{-i}\right)\right)\right]
$$

\footnotetext{
${ }^{14}$ Given that supplier 1 has mixed strategies under $\hat{b}^{1}, y^{i}(\hat{b}()$.$) already incorporates the expectation over$ supplier 1's mixed strategies. The same applies to $y^{1}(\hat{b}()$.$) later in this section.$
} 
Thus, the first term of (19) is also not changing if we switch from $b$ to $\hat{b}^{15}$ Hence, we find that overall the first term in (18) is not changing if we switch from $b$ to $\hat{b}$.

Now, let us consider supplier 1 and the second term in (18). Similar to (19) we have that

$$
\begin{aligned}
& \int_{\theta \in \Theta^{n}}\left[\tilde{x}^{1}(b(\theta))\left(v\left(q^{* 1}\left(\theta^{1}\right)\right)-\phi\left(q^{* 1}\left(\theta^{1}\right)\right)\right)+y^{1}(b(\theta))\right] \gamma(\theta) d \theta \\
= & \int_{\theta^{1} \in \Theta^{1}}\left[E_{-1}\left[\tilde{x}^{1}\left(b^{1}\left(\theta^{1}\right), b^{-1}\left(\theta^{-1}\right)\right)\right]\left(v\left(q^{* 1}\left(\theta^{1}\right)\right)-\phi\left(q^{* 1}\left(\theta^{1}\right)\right)\right)\right] \gamma^{1}\left(\theta^{1}\right) d \theta^{1} \\
& +\int_{\theta \in \Theta^{n}}\left[y^{1}(b(\theta))\right] \gamma(\theta) d \theta .
\end{aligned}
$$

Again, since by construction the distribution of suppliers' bids under $b$ is the same as under $\hat{b}$, the second term of $(20)$ is not changing if we switch from $b$ to $\hat{b}$, that is

$$
\int_{\theta \in \Theta^{n}}\left[y^{1}(b(\theta))\right] \gamma(\theta) d \theta=\int_{\theta \in \Theta^{n}}\left[y^{1}(\hat{b}(\theta))\right] \gamma(\theta) d \theta .
$$

Also as before, the distribution of bids coming from the other suppliers (suppliers other than 1 that is) is by construction the same under both $b$ and $\hat{b}$. However, since $\hat{b}^{1}$ is not necessarily equal to $b^{1}$, we cannot guarantee that for all $\theta^{1} \in \Theta$

$$
E_{-1}\left[\tilde{x}^{1}\left(b^{1}\left(\theta^{1}\right), b^{-1}\left(\theta^{-1}\right)\right)\right]=E_{-1}\left[\tilde{x}^{1}\left(\hat{b}^{1}\left(\theta^{1}\right), \hat{b}^{-1}\left(\theta^{-1}\right)\right)\right] \cdot{ }^{16}
$$

Nevertheless, from Lemma 3 it follows that the set of types $\theta^{1} \in \Theta$ for which

$$
E_{-1}\left[\tilde{x}^{1}\left(b^{1}\left(\theta^{1}\right), b^{-1}\left(\theta^{-1}\right)\right)\right] \neq E_{-1}\left[\tilde{x}^{1}\left(\hat{b}^{1}\left(\theta^{1}\right), b^{-1}\left(\theta^{-1}\right)\right)\right]
$$

has Lebesque measure zero. Thus, we can still guarantee that the first term of (20) is not changing if we switch from $b$ to $\hat{b}$.

Based on the above analysis we find that

$$
U^{0}(b)-U^{0}(\hat{b})=0
$$

that is, the expected utility for the buyer in equilibrium $b$ is the same as in $\hat{b}$. Furthermore, changing the bidding strategies of the other suppliers $(2, \ldots, n)$ one by one and applying the above analysis yields that also

$$
U^{0}(b)-U^{0}(\tilde{b})=0
$$

that is, the expected utility for the buyer in equilibrium $b$ is the same as in $\tilde{b}$.

\footnotetext{
${ }^{15}$ Given that supplier 1 has mixed strategies under $\hat{b}^{1}, E_{-i}[$.$] denotes the expectation taken over all type$ profiles $\theta^{-i}$ as well as the corresponding mixed strategies of supplier 1 .

${ }^{16}$ Given that supplier 1 has mixed strategies under $\hat{b}^{1}, E_{-1}[$.$] denotes the expectation taken over all type$ profiles $\theta^{-1}$ as well as the corresponding mixed strategies of supplier 1 .
} 


\section{References}

[1] Robert A. Adams, Calculus - A Complete Course, 5th ed., Addison Wesley Longman, Toronto, 2003.

[2] John Asker and Estelle Cantillon, Procurement When Both Price and Quality Matter, CEPR Discussion Paper, 6082, 2006.

[3] John Asker and Estelle Cantillon, Properties of Scoring Auctions, mimeo, 2007 (forthcoming RAND Journal of Economics).

[4] Lawrence M. Ausubel and Paul Milgrom, The Lovely but Lonely Vickrey Auction, in: Peter Cramton, Yoav Shoham and Richard Steinberg (Eds.), Combinatorial Auctions, MIT Press, Cambridge, 17-40, 2006.

[5] Yair Bartal, Rica Gonen and Noam Nisan, Incentive Compatible Multi Unit Combinatorial Auctions, in: Proc. of the 9th Conference on Theoretical Aspects of Rationality and Knowledge (TARK03), ACM Press, New York, 72-87, 2003.

[6] Martin Bichler, An Experimental Analysis of Multi-Attribute Auctions, Decision Support Systems, 29(3):249-268, 2000.

[7] Martin Bichler and Jayant Kalagnanam, Configurable Offers and Winner Determination in Multi-Attribute Auctions, European Journal of Operational Research, 160(2): 380$394,2005$.

[8] Fernando Branco, The Design of Multidimensional Auctions, Rand Journal of Economics, 28(1):63-81, 1997.

[9] Yeon-Koo Che, Design Competition Through Multidimensional Auctions, RAND Journal of Economics, 24(4):668-680, 1993.

[10] Ching-Hua Chen-Ritzo, Terry P. Harrison, Anthony M. Kwasnica and Douglas J. Thomas, Better, Faster, Cheaper: An Experimental Analysis of a Multiattribute Reverse Auction Mechanism with Restricted Information Feedback, Management Science, 51(12):1753-1762, 2005.

[11] Edward H. Clarke, Multipart Pricing for Public Goods, Public Choice, 11(1):17-33, 1971. 
[12] Peter Cramton, Yoav Shoham and Richard Steinberg (Eds.), Combinatorial Auctions, MIT Press, Cambridge, 2006.

[13] Esther David, Rina Azoulay-Schwartz and Sarit Kraus, An English Auction Protocol for Multi-Attributes Items, in: J. Padget, D. Parkes, N. Sadeh, O. Shehory, W. Walsh (Eds.), Agent Mediated Electronic Commerce: IV. Designing Mechanisms and Systems, vol. 2531:52-68, 2002 (a).

[14] Esther David, Rina Azoulay-Schwartz and Sarit Kraus, Bidders' Strategy for MultiAttribute Sequential English Auctions with a Deadline, in: Proc. of the Second International Joint Conference on Autonomous Agents and Multi-Agent Systems (AAMAS03), pp. 457-464, 2003.

[15] Esther David, Rina Azoulay-Schwartz and Sarit Kraus, Bidding in Sealed-Bid and English Multi-Attribute Auctions, Decision Support Systems, 42(2):527-556.

[16] Esther David, Rina Azoulay-Schwartz and Sarit Kraus, Protocols and Strategies for Automated Multi-Attributes Auctions, in: Proc. of the First International Joint Conference on Autonomous Agents and Multi-Agent Systems (AAMAS02), pp. 77-85, 2002 (b).

[17] Shahar Dobzinski, Noam Nisan and Michael Shapira, Truthful Randomized Mechanisms for Combinatorial Auctions, in: Proc. of the 38th Annual ACM Symposium on Theory of Computing (STOC06), ACM Press, New York, 644-652, 2006.

[18] Theodore Groves, Incentives in Teams, Econometrica, 41(4):617-631, 1973.

[19] Ron Holzman, Noa Kfir-Dahav, Dov Monderer and Moshe Tennenholtz, Bundling Equilibrium in Combinatorial Auctions, Games and Economic Behavior, 47:104-123, 2004.

[20] André I. Khuri, Advanced Calculus with Applications in Statistics, 2nd ed., Wiley, New Jersey, 2003.

[21] Vijay Krishna and Eliot Maenner, Convex Potentials with an Application to Mechanism Design, Econometrica, 69(4):1113-1119, 2001.

[22] Paul R. Milgrom, An Economist's Vision of the B-to-B Marketplace, Executive White Paper Perfect.com, 2000. 
[23] Rudolf Müller, Andrés Perea and Sascha Wolf, Weak Monotonicity and Bayes-Nash Incentive Compatibility, Games and Economic Behavior (forthcoming, available online), doi:10.1016/j.geb.2007.01.008, 2007.

[24] Noam Nisan, Bidding Languages for Combinatorial Auctions, in: Peter Cramton, Yoav Shoham and Richard Steinberg (Eds.), Combinatorial Auctions, MIT Press, Cambridge, 215-231, 2006.

[25] Noam Nisan and Armir Ronen, Computationally Feasible VCG Mechanisms, in: Proc. of the 2nd ACM Conference on Electronic Commerce (EC00), ACM Press, New York, 242-252, 2000.

[26] Michael Saks and Lan Yu, Weak Monotonicity Suffices for Truthfulness on Convex Domains, in: Proc. of the 6th ACM Conference on Electronic Commerce (EC05), ACM Press, New York, 286-293, 2005.

[27] Stefan Strecker and Stefan Seifert, Electronic Sourcing with Multi-Attribute Auctions, in: Proc. of the 37th Hawaii International Conference on System Sciences, 2004.

[28] Takayuki Suyama and Makoto Yokoo, Strategy/False-name Proof Protocols for Combinatorial Multi-Attribute Procurement Auction, Autonomous Agents and Multi-Agent Systems, 11(1):7-21, 2005.

[29] William Vickrey, Counterspeculation, Auctions, and Competitive Sealed Tenders, Journal of Finance, 16(1):8-37, 1961.

[30] Robert C. Wrede and Murray Spiegel, Schaum's Outline of Theory and Problems of Advanced Calculus, 2nd ed., McGraw-Hill, New York, 2002.

[31] K. Paul Yoon and Ching-Lai Hwang, Multiple Attribute Decision Making: An Introduction, Sage University Paper Series on Quantitative Applications in the Social Sciences, 07-104, 1995. 